\title{
Left Ventricular Assist Device Implantation in a Patient with Ventricular Pseudoaneurysm
}

\author{
Dejan Radakovic ${ }^{1} \quad$ Kiril Penov $^{1}$ Gülmisal Güder ${ }^{2} \quad$ Ivan Aleksic $^{10}$ \\ ${ }^{1}$ Department of Thoracic and Cardiovascular Surgery, Julius- \\ Maximilians-University Hospital Wuerzburg, Wuerzburg, Germany \\ 2 Internal Medicine I, Julius Maximilians University of Würzburg, \\ Wurzburg, Bayern, Germany

\begin{abstract}
Address for correspondence Ivan Aleksic, MD, PhD, Department of Thoracic and Cardiovascular Surgery, Julius Maximilians University of Würzburg, 97080, Wurzburg, Bavaria, Germany (e-mail: Aleksic_ı@ukw.de).
\end{abstract}

Thorac Cardiovasc Surg Rep 2022;11:e11-e13.

\begin{abstract}
Keywords

- heart

- heart failure

- LVAD

Background Left ventricular assist device (LVAD) implantation after contained LV rupture (pseudoaneurysm) represents a difficult surgical problem.

Case Description We describe the surgical approach for such a patient. The sewing ring was implanted utilizing a Dacron patch for reconstruction of the free wall, fibrotic LV wall remnants, and a Teflon strip giving additional support for cannula position and hemostasis. The patient had an uneventful recovery and is well 19 months after the procedure.

Conclusion LV pseudoaneurysm is not a contraindication for permanent LVAD implantation.
\end{abstract}

\section{Introduction}

Left ventricular assist device (LVAD) therapy has become an important strategy for patients with terminal heart failure. Implantation after contained rupture of the free wall of the left ventricle (pseudoaneurysm) is challenging and information scarce. ${ }^{1}$

LV free wall rupture following myocardial infarction (MI) is usually fatal and directly causes death in $\sim 8 \%$ of patients. ${ }^{2,3}$ The clinical course varies from cardiogenic shock to the slowly leaking type with pericardial effusion and hemodynamic instability. ${ }^{4}$

We describe the surgical approach for a patient with LV pseudoaneurysm and terminal heart failure who underwent LV reconstruction and LVAD implantation as destination therapy.

\section{Case Description}

A 70-year-old man was admitted with severe chest pain due to ST-elevation myocardial infarction. Emergent cardiac catheterization revealed occlusion of the circumflex artery and high-grade stenoses of the left anterior descending and the right coronary artery. Percutaneous coronary intervention with drug-eluting stent (PCI-DES) implantation restituted thrombolysis in myocardial infarction 2 flow in the circumflex. Left ventriculography demonstrated severely depressed ejection fraction (left ventricular ejection fraction [LVEF] 25\%) with an LV end-diastolic pressure of $29 \mathrm{~mm} \mathrm{Hg}$. Intervention on the other tributaries was postponed due to the clinical state with pulmonary edema. Ten days later, the right coronary artery was treated by PCI-DES, while the left anterior descending artery showed a stenosis of $50 \%$ only.

Twelve days after his second $\mathrm{PCI}$, the patient became hemodynamically unstable. Echocardiography showed new high-grade mitral regurgitation and pericardial tamponade with an LVEF of $20 \%$. Pericardiocentesis yielded a hemorrhagic effusion. Computed tomography scan revealed LV pseudoaneurysm (-Fig. 1A). The patient was intubated and developed refractory cardiogenic shock necessitating peripheral venoarterial extracorporeal membrane received

June 24, 2021

accepted after revision

September 13, 2021
DOI https://doi.org/

$10.1055 / \mathrm{s}-0041-1741554$. ISSN $2194-7635$.

\footnotetext{
(C) 2022. The Author(s).

This is an open access article published by Thieme under the terms of the Creative Commons Attribution-NonDerivative-NonCommercial-License, permitting copying and reproduction so long as the original work is given appropriate credit. Contents may not be used for commercial purposes, or adapted, remixed, transformed or built upon. (https://creativecommons.org/ licenses/by-nc-nd/4.0/) Georg Thieme Verlag KG, Rüdigerstraße 14, 70469 Stuttgart, Germany
} 


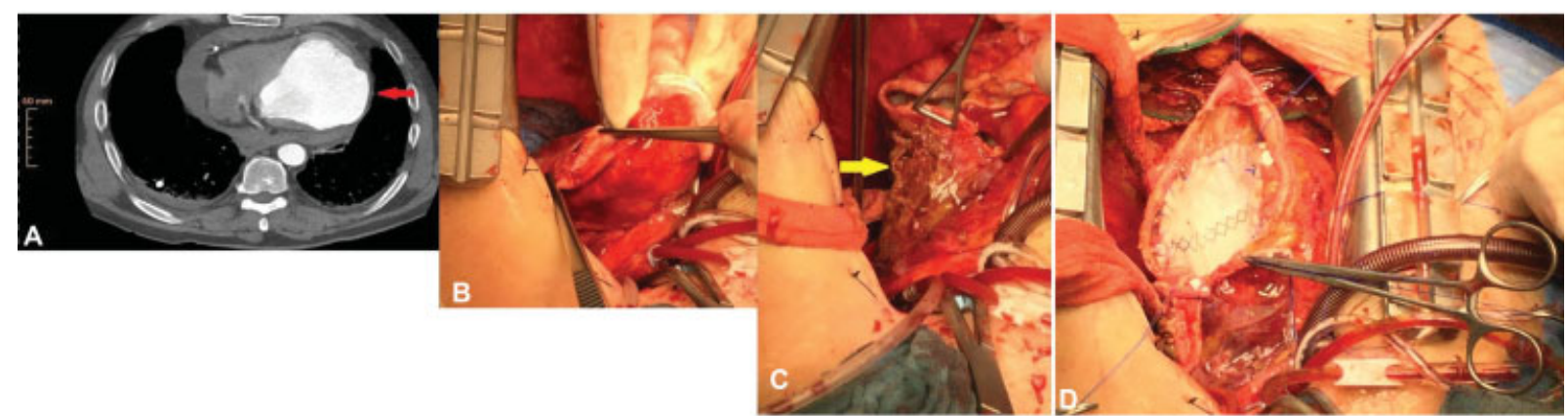

Fig. 1 (A) Left ventricular pseudoaneurysm on computed tomography (arrow). (B) Rupture site. (C) Extensive myocardial necrosis (arrow) after partial excision. (D) View of reconstructed left ventricle before incision for placement of the pump.

oxygenation (VA-ECMO) implantation via the right femoral vessels and Impella CP (Abiomed Europe, Aachen, Germany) for LV venting. Given the patient's age and clinical status, heart transplantation was not an option. We decided to implant a Medtronic HVAD (Medtronic, Dublin, Ireland) as destination therapy after reconstruction of the LV free wall after 5 days of combined VA-ECMO and Impella support.

Cardiopulmonary bypass was instituted via the peripheral VA-ECMO cannulas and an additional cannula in the superior vena cava.

The rupture site of the lateral wall was identified, and more than $50 \%$ of the LV muscle mass were estimated to be infarcted ( $\mathbf{F i g}$. 1B, C). The thinned wall was excised preserving a rim of fibrotic tissue for additional patch reinforcement. A Dacron patch was implanted with a 3-0 Prolene running suture (-Fig. 1D), a hole was excised with the HVAD suture ring serving as template. The exact position of the hole was selected after the introduction of a 22-mm Hegar dilator as described by Palmen et al..$^{5}$ To avoid creating an LV cavity too small for the inflow cannula and to prevent interference with the mitral valve apparatus, no Fontan stitch was applied in this situation. Patched 2-0 Ethibond U-sutures (Johnson \& Johnson Medical, Norderstedt, Germany) were placed in an inside-out fashion from the ventricular side of the Dacron patch, the remaining scar tissue and an additional Teflon strip for better hemostasis and further support for a stable position of the inflow cannula. Additional external Teflon strips were applied toward the base of the heart ( - Fig. 2A, B).
After completely uneventful recovery, the patient was discharged home on postoperative day 30 ( - Fig. 2C). Predischarge echocardiography demonstrated an end-systolic volume of $62 \mathrm{~mL}$ and an end-diastolic volume of $128.9 \mathrm{~mL}$. The patient has been followed up for 19 months now without any device-related complications.

\section{Discussion}

LVAD implantation has become an integral part of terminal heart failure treatment. Comorbidities or cardiac morphology may drastically increase complexity of the implant procedure and patient's risk.

The literature on LVAD implantation after contained free wall rupture is limited to few case reports. ${ }^{1,6,7}$ Two reasons precluded implantation of an extracorporeal LVAD with a left atrial inflow cannula in our patient: the contained rupture with a potential for late bleeding and a good quality of life postoperatively.

The Dor type LV reconstruction for LV aneurysms relies on the Fontan stitch for a purse-string effect at the transition zone of scarred and viable myocardium. We deliberately avoided the Fontan stitch to restore a LV cavity large enough to prevent inflow cannula obstruction due to small cavity size.

We tried to stabilize cannula position and achieve hemostasis by suturing the sewing ring to three layers: Dacron patch, scar tissue, and Teflon felt. The ideal position for the sewing ring was guided by Hegar dilators placed into the mitral valve orifice.

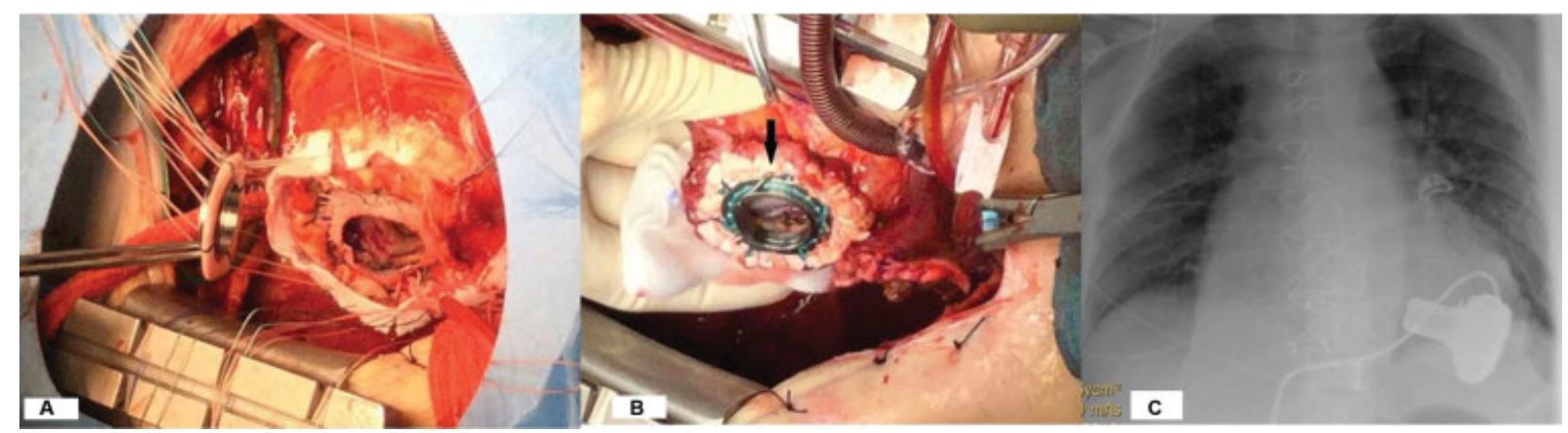

Fig. 2 (A) Ethibond U-sutures from ventricular side of Dacron patch, fibrous scar, and Teflon strip. (B) Final appearance before pump implantation. Note free access to the fixation screw (arrow). (C) Predischarge X-ray demonstrating proper inflow cannula position. 
The postoperative course and 19-month follow-up prove that the presence of an LV pseudoaneurysm is not a contraindication for LVAD implantation. It can be managed by patch plasty of the LV free wall. Any purse-string suture in the left ventricle should be omitted to avoid inflow cannula obstruction. Incorporation of scar tissue and Teflon strips into the ring fixation can contribute to hemostasis and a stable cannula position.

Since the Medtronic HVAD has been withdrawn from the market, recently, we believe that changes to the technique described herein are necessary for implantation of an Abbott HeartMate 3 (HM3) LVAD. With this device, sutures must be kept as short as possible to avoid interference with the locking mechanism between pump and sewing ring. Therefore, we would use three 3-0 Prolene running sutures with a Teflon felt as the innermost layer within the LV cavity followed by the Dacron patch and carefully trimmed scar tissue and suture these three layers to the sewing ring. An external Teflon strip above the scar tissue would probably interfere with the HM3 locking mechanism and should be omitted.

\section{Conflict of Interest}

None declared.

\section{References}

1 Kacer J, Lindovska M, Surovcik R, et al. Refractory cardiogenic shock due to extensive anterior STEMI with covered left ventricular free wall rupture treated with awake VA-ECMO and LVAD as a double bridge to heart transplantation - collaboration of three cardiac centres. Biomed Pap Med Fac Univ Palacky Olomouc Czech Repub 2015;159(04):681-687

2 Becker RC, Gore JM, Lambrew C, et al. A composite view of cardiac rupture in the United States National Registry of Myocardial Infarction. J Am Coll Cardiol 1996;27(06): 1321-1326

3 Reddy SG, Roberts WC. Frequency of rupture of the left ventricular free wall or ventricular septum among necropsy cases of fatal acute myocardial infarction since introduction of coronary care units. Am J Cardiol 1989;63(13):906-911

4 Padró JM, Mesa JM, Silvestre J, et al. Subacute cardiac rupture: repair with a sutureless technique. Ann Thorac Surg 1993;55(01): 20-23, discussion 23-24

5 Palmen M, Braun J, Beeres SLMA, Klautz RJM. Left ventricular assist device implantation in patients after left ventricular reconstruction. Interact Cardiovasc Thorac Surg 2016;23(06): 979-981

6 Firstenberg MS, Blais D, Crestanello J, et al. Long-term mechanical support for complex left ventricular postinfarct pseudoaneurysms. Heart Surg Forum 2009;12(05):E291-E293

7 Ha RV, Chiu P, Banerjee D, Sheikh AY. Dealing with a left ventricular pseudoaneurysm during assist device implant. Asian Cardiovasc Thorac Ann 2016;24(05):477-479 